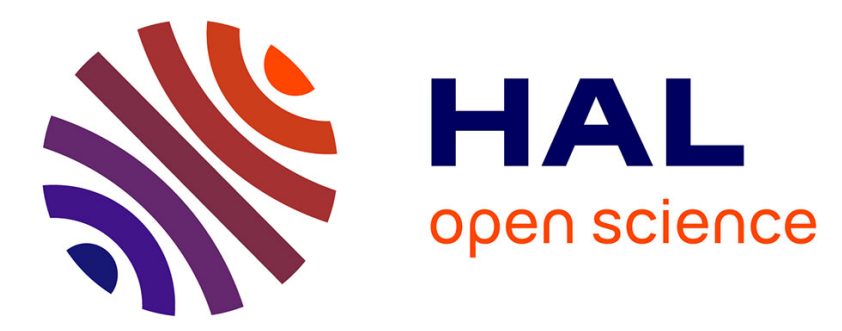

\title{
An innovative antisolvent precipitation process as a promising technique to prepare ultrafine rifampicin particles
}

Alessandra Vicosa, Jean-jacques Letourneau, Fabienne Espitalier, Maria-Inês Ré

\section{To cite this version:}

Alessandra Vicosa, Jean-jacques Letourneau, Fabienne Espitalier, Maria-Inês Ré. An innovative antisolvent precipitation process as a promising technique to prepare ultrafine rifampicin particles. JOURNAL OF CRYSTAL GROWTH, 2012, 342 (1), pp.80-87. 10.1016/j.jcrysgro.2011.09.012 hal01668417

\section{HAL Id: hal-01668417 https://hal.science/hal-01668417}

Submitted on 5 Sep 2018

HAL is a multi-disciplinary open access archive for the deposit and dissemination of scientific research documents, whether they are published or not. The documents may come from teaching and research institutions in France or abroad, or from public or private research centers.
L'archive ouverte pluridisciplinaire $\mathbf{H A L}$, est destinée au dépôt et à la diffusion de documents scientifiques de niveau recherche, publiés ou non, émanant des établissements d'enseignement et de recherche français ou étrangers, des laboratoires publics ou privés. 


\title{
An innovative antisolvent precipitation process as a promising technique to prepare ultrafine rifampicin particles
}

\author{
Alessandra Viçosa ${ }^{a, b, c}$, Jean-Jacques Letourneau ${ }^{a}$, Fabienne Espitalier ${ }^{a}$, Maria Inês Ré ${ }^{a, *}$ \\ ${ }^{a}$ Université de Toulouse, Mines Albi, CNRS, Centre RAPSODEE, Campus Jarlard, F-81013 Albi CT cedex 09, France \\ ${ }^{\mathrm{b}}$ Laboratório de Tecnologia Farmacêutica, Complexo Tecnológico de Medicamentos, Fundação Oswaldo Cruz, Av. Comandante Guaranys 447 \\ Jacarepagua 21041-250, Rio de Janeiro-RJ, Brazil \\ ' Instituto de Macromoléculas, Universidade Federal do Rio de Janeiro, Centro de Tecnologia, Av. Horacio Macedo 2030, Bloco J, Ilha do Fundão 21945-598, Rio de Janeiro—RJ, Brazil
}

\begin{abstract}
A B S T R A C T
Many existing and new drugs fail to be fully utilized because of their limited bioavailability due to poor solubility in aqueous media (BCS drug classes II and IV). In this work, for accelerating dissolution of this kind of poorly water-soluble drugs, an antisolvent precipitation method that does not require the use of conventional volatile organic solvents is proposed. To demonstrate this technique, ultrafine particles of rifampicin were prepared using a room temperature ionic liquid (1-ethyl 3- methyl imidazolium methyl-phosphonate) as an alternative solvent and a phosphate buffer as an antisolvent.

Rifampicin solubility was measured in various solvents (1-ethyl 3-methyl imidazolium methylphosphonate, water and phosphate buffer), showing the RTIL good solvency for the model drug: rifampicin solubility was found to be higher than $90 \mathrm{mg} / \mathrm{g}$ in RTIL at $30^{\circ} \mathrm{C}$ and lower than $1 \mathrm{mg} / \mathrm{g}$ in water at $25^{\circ} \mathrm{C}$. Additionally, it was demonstrated that introduction of rifampicin solution in 1-ethyl 3- methyl imidazolium methyl-phosphonate into the aqueous solution antisolvent can produce particles in the submicron range with or without hydroxypropyl methylcellulose as the stabilizer. The ultrafine particles (280-360 $\mathrm{nm}$ ) are amorphous with enhanced solubility and faster dissolution rate. To our knowledge, this is the first published work examining the suitability of using RTILs for ultrafine drug nanoparticles preparation by an antisolvent precipitation process.
\end{abstract}

Keywords:

A1. Antisolvent precipitation

A1. Bioavailability enhancement

B1. Drug nanoparticles

B1. Poorly water-soluble drug

B1. Rifampicin

B1. Room temperature ionic liquids

\section{Introduction}

It is estimated that a significant proportion of drugs in the market are poorly soluble in water, and it is expected that this will be even more pronounced in the future $[1,2]$. Formulation of poorly water-soluble drugs is a challenge in pharmaceutical field because typical problems associated with this class of compounds are a too low oral bioavailability and erratic absorption [3-5]. Among the many factors that influence oral drug absorption, solubility in water and physiological media as well as intestinal permeability are keys for determining the fraction of dose absorbed.

Dissolution is considered the rate limiting step to drug absorption for some poorly soluble drugs, especially those with high intestinal permeability. Dissolution kinetics may be increased by reducing the particle size to increase the surface area [6], by coating drug particles with hydrophilic surfactants to enhance wetting and solvation by intestinal fluids [7-9], formation of solid dispersions $[10,11]$ and the transformation of crystalline drug to amorphous state [12].

\footnotetext{
* Corresponding author. Tel.: +33 563493299; fax: +33 563493025

E-mail address: maria-ines.re@mines-albi.fr (M. Inês Ré).
}

With regard to the Noyes-Whitney and Ostwald-Freundlich equations, size reduction can offer increased dissolution and solubility characteristics [13]. Many approaches have been attempted to reduce particle size, including mechanical micronization [14], supercritical fluid technique [15] and controlled antisolvent precipitation [16].

The mechanical micronization methods need high-energy input and show some disadvantages in practice such as electrostatic effects, broad particle size distributions, thermal degradation, contamination and reproducibility problems among different batches [17]. The reduction of particle size based on the use of supercritical antisolvents (SAS) has been suggested during the past years as an alternative to traditional liquid antisolvent processes. In this process, supercritical fluids substitute the liquid antisolvent to induce the precipitation of microparticles with controlled diameter and particle size distribution. Supercritical fluid technique is believed to be an attractive method for size reduction, providing particles with narrow size distribution. However, it also has the limitations of low yield and high equipment cost [18].

Antisolvent precipitation process is a promising technique to prepare ultrafine drug particles [17,19]. The basic principle is that the drug is dissolved in a solvent; the solvent solution is then mixed with an antisolvent (in which the drug is insoluble). The drug 
precipitates as a consequence of the change of supersaturation caused by mixing the solution and the antisolvent. The key for producing ultrafine particles by antisolvent precipitation is to create conditions that favor very rapid particle formation and little or no particle growth. The technique presents some advantages, in that it is a straightforward method, rapid and easy to perform. It has been successfully used to prepare several drugs, such as budesonide [20], danazol [21], beclomethasone dipropionate [18], prednisolone [22], atorvastatin [23], griseofulvin and fenofibrate [24].

However, one technological limitation associated with this technology is that the drug must be soluble enough in a solvent. New drugs (for the most part) lack sufficient solubility in water or even in organic solvents. Typical organic solvents that have been used are dimethyl sulfoxide (DMSO) or acetone. Their use is not fully recommended due to reasons such as solvent toxicity, limitation of a very low residual content in the precipitated solid and environmental aspects (solvent/antisolvent separation and solvent recycling).

RTILs (room temperature ionic liquids) are organic salts, which are liquid at ambient room temperature. They are composed of ions comprising of a relatively large asymmetric organic cation (e.g. alkyl pyridinium, dialkyl imidazolium ions) and an inorganic or organic anion (e.g. halide, hexafluorophosphate, tetrafluoroborate and ions based on fluorinated amides). The asymmetry of the cation is responsible for low lattice energies of RTILs and their liquid state at ambient conditions. The overall properties of the RTILs depend on those of their substituent cations and anions, and RTILs with a wide variation in acidicity, basicity, hydrophilicity/ hydrophobicity and water miscibility have been reported [25].

Recently, RTILs were found to be good solvents for some model poorly water-soluble drugs such as albendazole and danazol [26]. In this work, an RTIL based on 1-ethyl-3-methylimidazolium cation was firstly investigated as an alternative solvent for rifampicin, a poorly water-soluble drug. Rifampicin, $\mathrm{C}_{43} \mathrm{H}_{58} \mathrm{~N}_{4} \mathrm{O}_{12}$, is a class II drug of Biopharmaceutical Classification System (BCS), where rate and extent of dissolution are critical for optimum bioavailability [27-29]. A second objective of this work was to prepare rifampicin ultrafine powder via antisolvent precipitation process in order to improve its solubility and dissolution kinetics. An aqueous solution has been chosen as an antisolvent.

\section{Materials and methods}

\subsection{Materials}

The raw rifampicin (coded RIFA) was obtained from Luohe Nanjiecun Pharmaceutical (Group Pharmacy China) and used without further purification.

Two reference polymorphic samples of rifampicin (forms I and II) were provided by Farmanguinhos Pharm. Laboratory, coded RIFA1 and RIFA2, respectively. The reference samples are commercial samples that were selected and characterized (X-ray diffraction, differential scanning calorimetry and hot-stage microscopy) for Farmanguinhos research use.

RTIL 1-ethyl 3-methyl imidazolium methyl-phosphonate ( > 98\% pure) was obtained from Solvionic (Toulouse, France), its melting point is lower than $255 \mathrm{~K}$ and it was used as received.

The structural formulas of both rifampicin and 1-ethyl 3-methyl imidazolium methyl-phosphonate are given in Fig. 1.

Sodium hydroxide $(\mathrm{NaOH})$ and potassium dihydrogen phosphate $\left(\mathrm{KH}_{2} \mathrm{PO}_{4}\right)$ were of analytical grade, obtained commercially and used to prepare the phosphate buffer ( $\mathrm{pH}$ 6.8). Hydroxypropyl methylcellulose (HPMC) of low viscosity grade was used as an additive in the process.

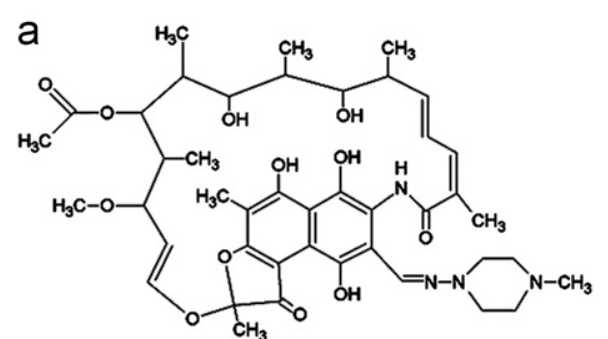

b

Fig. 1. Chemical structure of drug and solvent used in this study. (a) drug model (rifampicin); (b) solvent RTIL (cation: 1-ethyl-3-methylimidazolium; anion: methylphosphonate).

\subsection{Methods}

\subsubsection{Determination of solubility}

The solubility of rifampicin in different solvents (water, RTIL, phosphate buffer with a $\mathrm{pH}$ equal to 6.8 ) or mixtures of solvents (mixture of RTIL and phosphate buffer) was determined according to the following procedure: agitated solutions, containing an excess of solid, are maintained at controlled temperature $\left(30 \pm 2{ }^{\circ} \mathrm{C}\right.$ for pure IL and $25 \pm 2{ }^{\circ} \mathrm{C}$ for the others) and atmospheric pressure. According to the experiment, after $24 \mathrm{~h}$ (for pure solvents) or $56 \mathrm{~h}$ (mixture of solvents RTIL/phosphate buffer), the agitation was stopped to settle the mixture and the supernatant was filtered through a $0.2 \mu \mathrm{m}$ membrane filter (Acrodisc PSF GHP, Millipore, Bedford, MA, US). An aliquot of the filtered liquid was adequately diluted and assayed spectrophotometrically (UV-vis Diode-Array spectrophotometer HP 8452A) at wavelengths of $333 \mathrm{~nm}$ (for aqueous phase) and $482 \mathrm{~nm}$ (for RTIL) to evaluate the amounts of drug dissolved. Different RTIL/phosphate buffer ratios $(1: 5 ; 1: 10 ; 1: 20$ and $1: 100 \% \mathrm{w} / \mathrm{w})$ were used. Experiments were conducted in duplicate.

Preliminary experiments were carried out to select the antisolvent for rifampicin to be used in the antisolvent process. Water was the first choice, however the results obtained confirmed the chemical degradation of this molecule (very sensitive to acidic $\mathrm{pH}$ conditions) in an RTIL/water system. Phosphate buffer ( $\mathrm{pH}$ 6.8) was thus selected in the final phase of the previous experiments (not shown here).

The solubility data were used to estimate the theoretical efficiency of the process as a function of RTIL/phosphate buffer ratios. Theoretical solid efficiency is the theoretical solid yield of precipitation based on the drug solubility in the liquid media, calculated from the ratio of weight particles and the initial weight of solute.

\subsubsection{Preparation of ultrafine rifampicin}

The experimental procedure for the preparation of ultrafine rifampicin is schematized in Fig. 2. Briefly, raw rifampicin (RIFA) was dissolved in RTIL at $30 \pm 2{ }^{\circ} \mathrm{C}$ to give an RTIL solution containing $90 \mathrm{mg}$ rifampicin/g solution. Afterwards, this solution was poured into a stirred phosphate buffer solution at $25 \pm 2{ }^{\circ} \mathrm{C}$ ( $1 \mathrm{~L}$ vessel). Particles were formed immediately. After stirring for a pre-determined time $(0.5 \mathrm{~h}$ or $4 \mathrm{~h})$, the solid particles were recovered by centrifugation and washed with phosphate buffer. The procedure was repeated and the rifampicin particles were then rinsed with deionized water in the final washing step. The solid was dried in vacuum oven at $50{ }^{\circ} \mathrm{C}$ for $24 \mathrm{~h}$.

In some experiments, hydroxypropyl methylcellulose (HPMC) was used as a hydrophilic agent to control the particle morphology and inhibit particle agglomeration. Briefly, HPMC was added to $100 \mathrm{~mL}$ phosphate buffer (0.1 or $0.5 \mathrm{wt} \%$ ). 


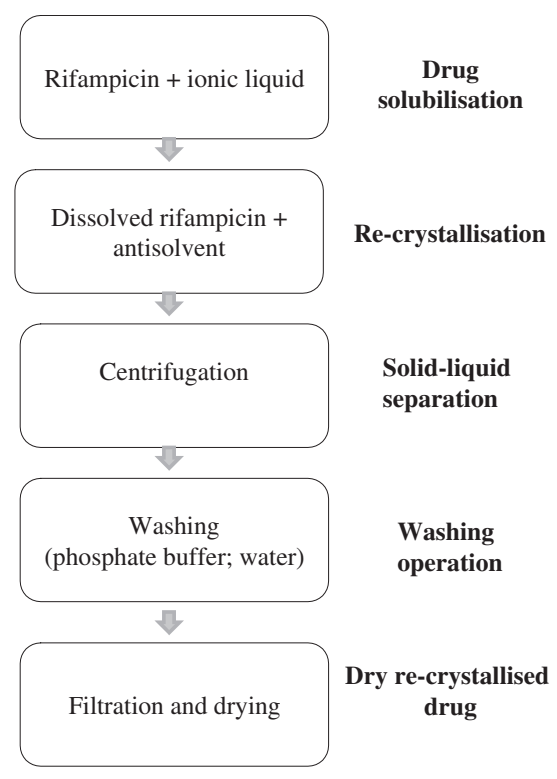

Fig. 2. Scheme of the experimental process to prepare ultrafine rifampicin.

\subsubsection{Characterization of products}

2.2.3.1. Particle size analysis. Particle size analysis was applied to RIFA sample by laser diffractometry using a Mastersizer 2000 (Malvern Instruments, UK). Photon correlation spectroscopy (PCS) using a Zetasizer $3000 \mathrm{HS}$ (Malvern Instruments, UK) was used to measure the particle size of rifampicin powder after processing. Before analysis, RIFA and precipitated samples were adequately diluted (mineral oil or $0.1 \mathrm{w} / \mathrm{v} \%$ HPMC in water) to obtain an appropriate obscuration.

2.2.3.2. Scanning electron microscopy (SEM). SEM analysis was used to examine the surface structure and shape of the drug particles and also to verify qualitatively the particle size obtained from the particle size analysis. An Environmental Scanning Electron Microscope-Field Emission Gun microscope (Philips XL30 ESEM-FEG, FEI Company) was used to record SEMpictures. The samples were fixed on an SEM stub using doublesided adhesive tape and coated with a thin layer of gold in a SC7640 sputtering device (Polaron).

2.2.3.3. X-ray diffraction studies (XRD). Powder X-ray diffraction measurements were performed by using a Philips X'Pert X-ray diffractometer (PANanalytical). $\mathrm{CuK}_{\alpha}$ radiation with a wavelength of $1.5425 \AA$ at $40 \mathrm{~mA}$ and $40 \mathrm{kV}$ was used. The diffraction patterns were collected in an angular range of $5^{\circ}$ to $60^{\circ}-2 \theta$ with a step size of $0.2^{\circ}$. The time counting was $15 \mathrm{~s}$ per step.

2.2.3.4. Differential scanning calorimetry (DSC). The phase transition of samples was analyzed by differential scanning calorimeter (Q200, TA Instruments) operating in a temperature range of $20-300^{\circ} \mathrm{C}$ at a heating rate of $10^{\circ} \mathrm{C} / \mathrm{min}$. A dry nitrogen purge of $80 \mathrm{~mL} / \mathrm{min}$ was employed in the process. Calibration of the instrument with respect to temperature and enthalpy was achieved using high purity standard of indium.

2.2.3.5. Purity. Fifty milligrams of rifampicin was solubilized in $20 \mathrm{~g}$ of DMSO (initial concentration $2.5 \mathrm{mg} / \mathrm{g}$ ). The sample was adequately diluted and assayed spectrophotometrically (UV-vis Diode-Array spectrophotometer HP 8452A) at wavelength of $482 \mathrm{~nm}$ to evaluate the amounts of drug dissolved. All experiments were carried out in duplicate. The purity is the ratio between the measured concentration and the initial concentration.

2.2.3.6. Water content. The water content was determined using Infrared balance (LJ16, Mettler) at $100{ }^{\circ} \mathrm{C}$ until constant weight was achieved.

2.2.3.7. HPLC instrumentation and conditions. The HPLC system comprised an Agilent 1100 with auto-sampling system, column oven, integrated solvent and sample management configuration (Agilent). The mobile phase consisted of a 25.5:17.5:5:1:1 (v/v) mixture of water, acetonitrile, phosphate buffer, sodium perchlorate $0.5 \mathrm{M}$ and citric acid $1 \mathrm{M}$ solutions. The column USP category L7 (Prontosil 120-5-C8-SH $5 \mu \mathrm{m} 100 \mathrm{~mm} \times 4.6 \mathrm{~mm}$ ) was maintained at room temperature and equilibrated for minimum of $60 \mathrm{~min}$ with the analytical mobile phase before injection. The injection volume was $50 \mu \mathrm{L}$, and the mobile phase was pumped isocratically at a flow rate of $1.5 \mathrm{ml} / \mathrm{min}$. The effluent was monitored at $280 \mathrm{~nm}$.

2.2.3.8. Dissolution testing. Dissolution of drugs was performed according to the USP XXV type II (paddle) method (DT60 dissolution apparatus, ERWEKA). The rotation speed of paddle was set to $100 \mathrm{rpm}$ and the bath temperature was kept at $37.0 \pm 0.5^{\circ} \mathrm{C}$. Fifty milligrams of drug powder was put into the vessel containing $900 \mathrm{ml}$ of water. At specific intervals, $5 \mathrm{ml}$ aliquot of the dissolution medium was sampled, filtered (pore size $0.45 \mu \mathrm{m}$ ) and assayed spectrophotometrically (UV-vis DiodeArray spectrophotometer HP $8452 \mathrm{~A}$ ) at wavelength of $333 \mathrm{~nm}$ to evaluate the amounts of drug dissolved.

\section{Results and discussion}

\subsection{Characterization of raw rifampicin}

A sample of commercially available powder of rifampicin (coded RIFA) and two reference samples of rifampicin (polymorphic forms I and II coded RIFA1 and RIFA2, respectively) were characterized by DSC and X-ray diffraction analysis. The analysis of the two reference samples aims at facilitating the chemical identification of the RIFA sample, which was used in the antisolvent precipitation process. This commercially available powder has a purity of $99.11 \pm 0.95 \%$ and a water content of $1.82 \mathrm{w} / \mathrm{w} \%$, which have been experimentally determined.

Fig. 3 shows DSC thermograms for all rifampicin samples. The DSC thermogram of RIFA shows an endothermic process close to $190{ }^{\circ} \mathrm{C}$ and a small exotherm around $208{ }^{\circ} \mathrm{C}$ (maximum peak temperatures). A second exotherm probably due to decomposition is observed at $259^{\circ} \mathrm{C}$. Although glass transition $(\mathrm{Tg})$ is not detectable in the $y$-axis scale values of the graph compared to the other peak temperatures, a $\mathrm{Tg}$ occurred around $138^{\circ} \mathrm{C}$. It could correspond to the glass transition that occurred in an amorphous region of RIFA sample.

Moreover, the general appearance of the DSC thermograms of the two reference samples was the same as that for samples of polymorphic forms I and II described in the literature, although slight differences in peak sizes and positions were observed [30,31]: RIFAF1 (form I) directly decomposes at $267^{\circ} \mathrm{C}$, whereas RIFAF2 (form II) shows a melting endotherm at $196^{\circ} \mathrm{C}$ immediately followed by re-crystallization to form I at $207^{\circ} \mathrm{C}$, which is a characteristic of solid-liquid-solid transition and finally decomposes at $255^{\circ} \mathrm{C}$.

In order to further confirm the physical state of the raw material and the reference samples, XRD analysis was performed. Although the XRD patterns were collected in an angular range of $5^{\circ}$ to $60^{\circ}-2 \theta$, the angular range of $5^{\circ}$ to $30^{\circ}$ was sufficient to 


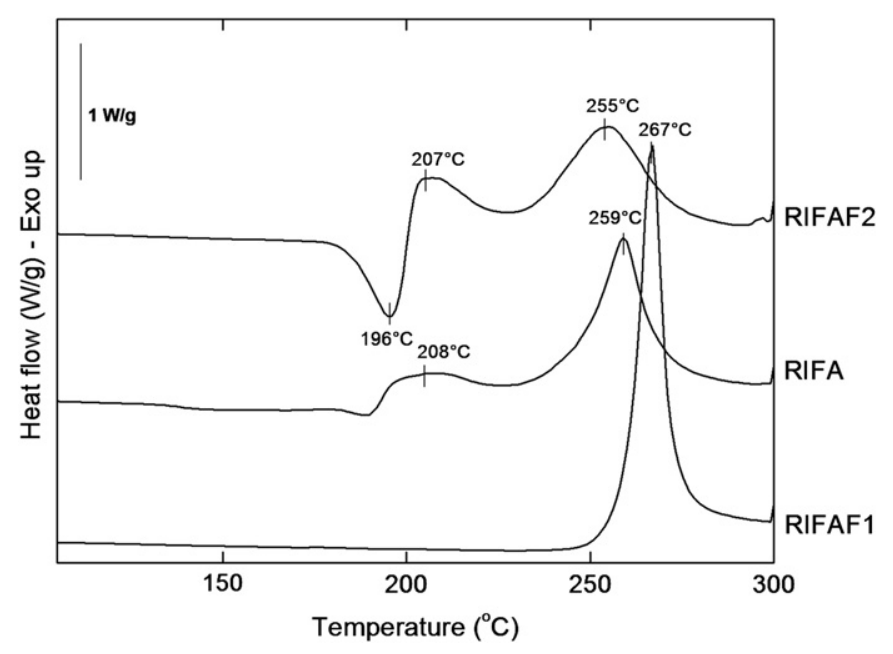

Fig. 3. DSC thermograms of the raw rifampicin (RIFA) compared to reference rifampicin samples, form I (RIFAF1) and form II (RIFAF2).

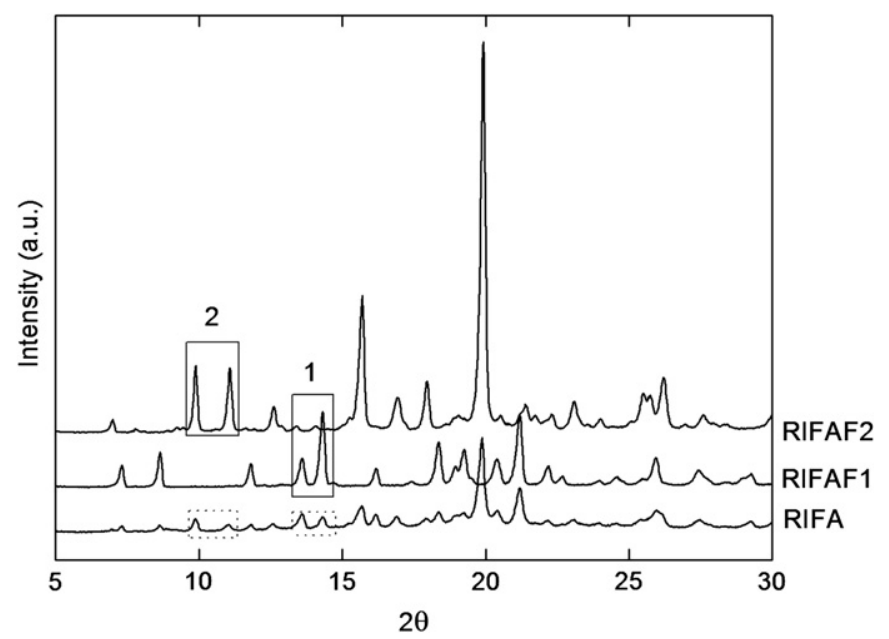

Fig. 4. X-ray diffraction patterns of form I (RIFAF1), form II (RIFAF2) and the raw rifampicin sample (RIFA).

allow the identification of characteristic peaks of forms I and II (see Fig. 4). Characteristic peaks for form I were observed at $13.65^{\circ}$ and $14.35^{\circ} 2 \theta$, whereas form II exhibited diffraction peaks at $9.93^{\circ}$ and $11.10^{\circ} 2 \theta$. It can be seen that these four peaks were also present in the XRD patterns of the RIFA sample.

Based on the literature data and on the DSC and XRD analysis shown here for the RIFA sample, we concluded that the powder used in the antisolvent precipitation study was a mixture of forms I, II and amorphous rifampicin. However, it was not possible to discern the presence of an amorphous region in the crystalline polymorphous mixture (forms I and II) from XRD analysis (no broad halo). The crystalline phases probably represent the most part of this sample.

\subsection{Solubility studies}

\subsubsection{Solvency of RTIL}

The solubility of the raw rifampicin in the chosen RTIL is higher than $90 \mathrm{mg}$ rifampicin/g RTIL. However, the equilibrium solubility data could not be determined due to the gel occurrence for higher rifampicin concentrations in this RTIL solvent.

As anticipated, the RTIL proved to be a good solvent for rifampicin, compared to other solvents (literature data summarized in Table 1).
Table 1

Solubility of rifampicin in different solvents.

\begin{tabular}{|c|c|c|c|c|}
\hline Solvent & $\begin{array}{l}\text { Solubility } \\
(\mathrm{mg} / \mathrm{ml})\end{array}$ & Classification & Reference & Obs \\
\hline Soybean oil & 45.4 & - & [29] & At $30{ }^{\circ} \mathrm{C}$ \\
\hline $\begin{array}{l}\text { Isopropyl } \\
\text { myritate }\end{array}$ & 10.0 & - & [29] & At $30^{\circ} \mathrm{C}$ \\
\hline $\begin{array}{l}\text { Isopropyl } \\
\text { palmitate }\end{array}$ & 6.6 & - & [29] & At $30^{\circ} \mathrm{C}$ \\
\hline Ethyl oleate & 6.3 & - & [29] & At $30^{\circ} \mathrm{C}$ \\
\hline Tween 80 & 22.0 & - & [29] & At $30{ }^{\circ} \mathrm{C}$ \\
\hline Tween 20 & 20.1 & - & [29] & At $30^{\circ} \mathrm{C}$ \\
\hline Methyl alcohol & 60 & Soluble & {$[32,33]$} & At room temp. \\
\hline Ethyl acetate & 40 & Soluble & {$[32,33]$} & At room temp. \\
\hline $\begin{array}{l}N \text {-methyl } \\
\text { 2-pyrrolidone }\end{array}$ & 80 & - & [32] & At room temp. \\
\hline Dichloromethane & 60 & - & {$[32]$} & At room temp. \\
\hline
\end{tabular}

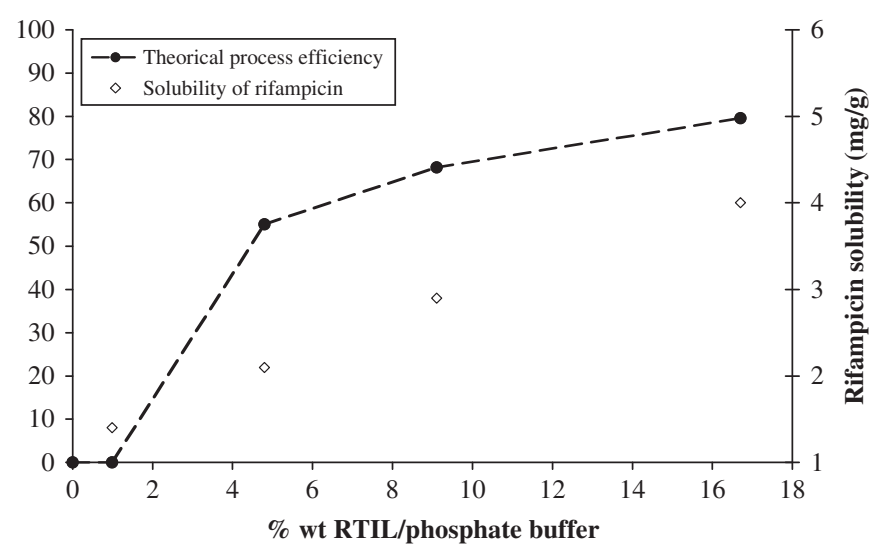

Fig. 5. Solubility of rifampicin in RTIL/phosphate buffer solution as a function of antisolvent content and corresponding percentage of rifampicin that re-crystallizes from the initial rifampicin/RTIL solution (theoretical process efficiency, \%).

For drug dissolution to occur, the energy generated by solute-solvent interactions must be greater than the energy needed for solute and solvent to separate. The levels of solubility observed for this drug indicate appreciable rifampicin/(1-ethyl 3-methyl imidazolium methyl-phosphonate) interactions.

\subsubsection{Solubility of raw rifampicin in different RTIL/phosphate buffer} combinations

Fig. 5 depicts the results obtained in the solubility experiments, including the theoretical solid efficiency. It can be seen that the solubility of rifampicin decreases rapidly with the addition of phosphate buffer to the RTIL/phosphate buffer solution. Solubility of rifampicin in phosphate buffer was found to be around $1 \mathrm{mg} / \mathrm{g}$. It can also be seen that a RTIL/phosphate buffer of $16.7 \%$ is still favorable for the precipitation process, resulting in a recovery of $79.6 \%$ of rifampicin based on the drug solubility in the liquid media.

\subsection{Antisolvent crystallization}

\subsubsection{Process efficiency}

Experiments of antisolvent precipitation of rifampicin were carried out with different solvent-to-antisolvent mass ratios and different crystallization times, as summarized in Table 2 . The process condition named "instantaneous" corresponds to the experience 
Table 2

Antisolvent crystallization process conditions and product characteristics.

\begin{tabular}{|c|c|c|c|c|c|}
\hline \multirow{2}{*}{$\begin{array}{l}\text { Experiment } \\
\text { number }\end{array}$} & \multirow{2}{*}{$\begin{array}{l}\text { Purity } \\
\text { (w/w\%) }\end{array}$} & \multirow{2}{*}{$\begin{array}{l}\text { Water } \\
\text { content } \\
(\%)\end{array}$} & \multicolumn{3}{|c|}{ Process conditions } \\
\hline & & & $\begin{array}{l}\text { RTIL/ } \\
\text { phosphate } \\
\text { buffer }\end{array}$ & $\begin{array}{l}\text { HPMC } \\
(\%)\end{array}$ & $\begin{array}{l}\text { Crystallization } \\
\text { time before } \\
\text { sampling (h) }\end{array}$ \\
\hline 1 & $93.1 \pm 3.4$ & 6.9 & $1: 10$ & 0 & 0.5 \\
\hline 2 & $107.7 \pm 3.8$ & 8.9 & $1: 10$ & 0 & 4 \\
\hline 3 & $96.1 \pm 2.0$ & 7.0 & $1: 5$ & 0 & 4 \\
\hline 4 & $98.4^{*}$ & 7.5 & $1: 5$ & 0 & 4 \\
\hline 5 & $95.1 \pm 1.7$ & 6.8 & $1: 5$ & 0 & instantaneously \\
\hline 6 & $90.7 \pm 3.0$ & 6.0 & $1: 5$ & 0.1 & 4 \\
\hline 7 & $97.8 \pm 0.9$ & 11.0 & $1: 5$ & 0.5 & 4 \\
\hline
\end{tabular}

$$
\text { * } n=1 \text {. }
$$

where the crystals were washed and dried immediately after pouring the rifampicin/RTIL solution into the antisolvent.

Theoretically, at the end of the experiments carried out in this study, a recovery of $79.6 \%$ and $68.2 \%$ of rifampicin could be expected for the ratio RTIL:phosphate buffer of $1: 5$ and $1: 10$, respectively (kinetics not considered). The lower efficacy for a process carried out with a higher amount of antisolvent (RTIL:phosphate buffer of 1:10) can be explained by a dilution effect, considering that the rifampicin solubility in both ratios is very close.

At each step of the process, the amounts of the solid or liquids used or produced were strictly controlled (precipitated solids, solution preparations, empty vials and filters). The mass losses due to the transfer operations of solid or liquids during the antisolvent crystallization process were quantified. The closure of the mass balance of rifampicin (total amount of solid + solute recovered/amount of solid dissolved) in all of the experiments was greater than $86 \%$.

In practice, at the end of the experiments, a solid efficiency of $40-50 \%$ as the final product of the antisolvent precipitation process was obtained. The difference compared to the maximum theoretical recovery (68.2-79.6\%) was mainly due to the drug loss during the washing steps.

\subsubsection{Product characterization}

Purity and water content. The powder obtained is characterized by purity ranging from $93 \%$ to $108 \%$, very close to the United States Pharmacopea recommended range of 95-103\% (USP 29 monograph). The water content ranged from $6 \%$ to $11 \%$ (dry basis) as shown in Table 2, which is much higher than that in the raw rifampicin before processing (1.82\%) probably due to the operating conditions during the drying step.

Morphology and particle size. Fig. 6 shows SEM images of rifampicin powders. The precipitated powder (Figs. 6a and b) is constituted by micron-large aggregates. However, the SEM images show that the aggregates are composed of a large number of individual submicron particles. Particle size and polydispersity index of these individual submicron particles were determined by photon correlation spectroscopy. Average particle sizes of precipitated powders obtained from Experiment numbers 3, 6 and 7 (see Table 2) were found to be 283,310 and $353 \mathrm{~nm}$, respectively. Polydispersity index were found to be 0.19 for Experiment 3, 0.82 for Experiment 6 and 0.45 for Experiment 7.

Before processing, the average particle size of raw rifampicin (Fig. 6c) was about $325 \mu \mathrm{m}$ with a wide particle size distribution $(40-712 \mu \mathrm{m})$. Evidently, the particle size of the nanosized rifampicin is significantly smaller and more uniform than that of the raw drug, which should be more beneficial for enhancing bioavailability. a

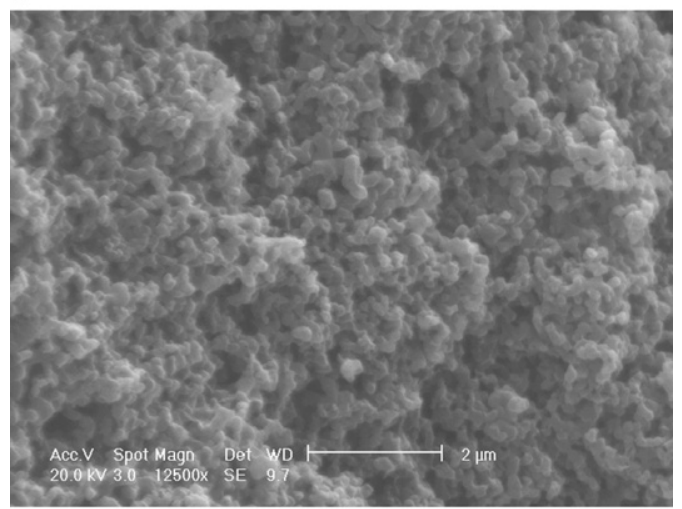

b

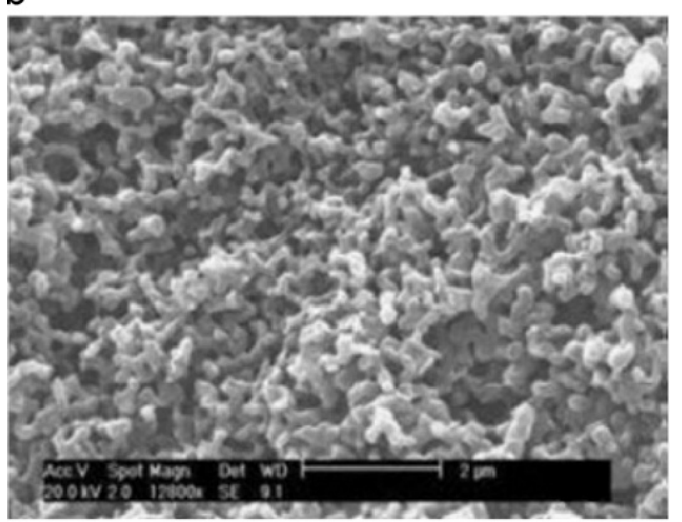

C

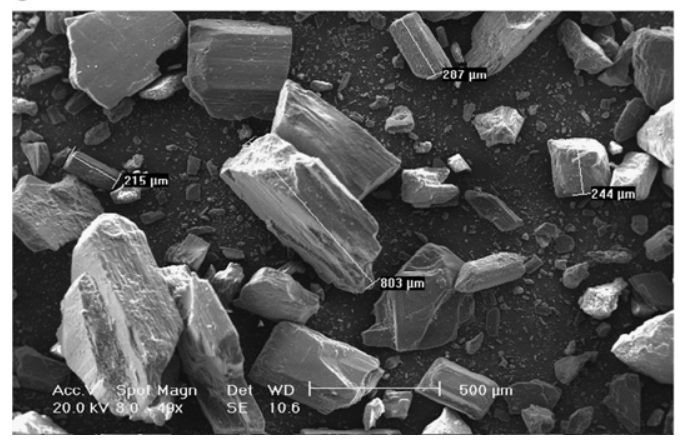

Fig. 6. SEM images of rifampicin powders. (a) Precipitated powder obtained without any stabilizer (experiment 1); (b) precipitated powder obtained with a stabilizing agent in the antisolvent (experiment 2); (c) RIFA (raw rifampicin).

$X R D$ analysis. The solid structures of the nano-sized rifampicin were studied by means of XRD analysis. The resulting profiles are shown in Fig. 7. The crystalline peaks were not found in the diffraction patterns of the samples, demonstrating that all precipitated nanosized rifampicin samples were amorphous. It is believed that poorly water-soluble pharmaceuticals with lower crystallinity and smaller size usually exhibit higher dissolution rate and bioavailability. Accordingly, the decrease in crystallinity and the size reduction of the precipitated particles are expected to improve the rifampicin dissolution rate and bioavailability.

\subsubsection{Dissolution studies}

Aqueous dissolution rates have been correlated to the in vivo performance of drug delivery systems. The in vitro release profiles of raw rifampicin and nanosized rifampicin are compared in Fig. 8. 


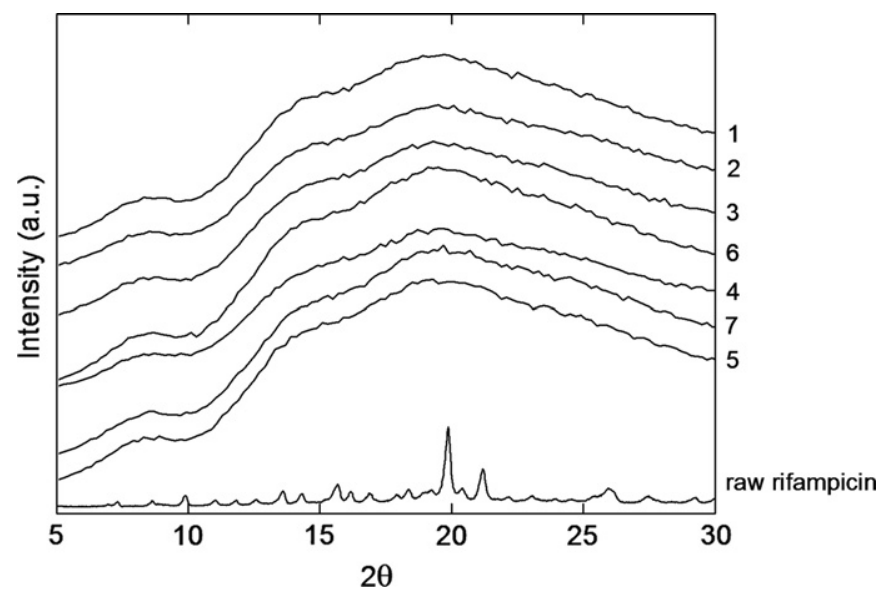

Fig. 7. Powder X-ray diffractograms of rifampicin powders. RIFA: raw rifampicin; experiments 1 to 7 precipitated from RIFA.

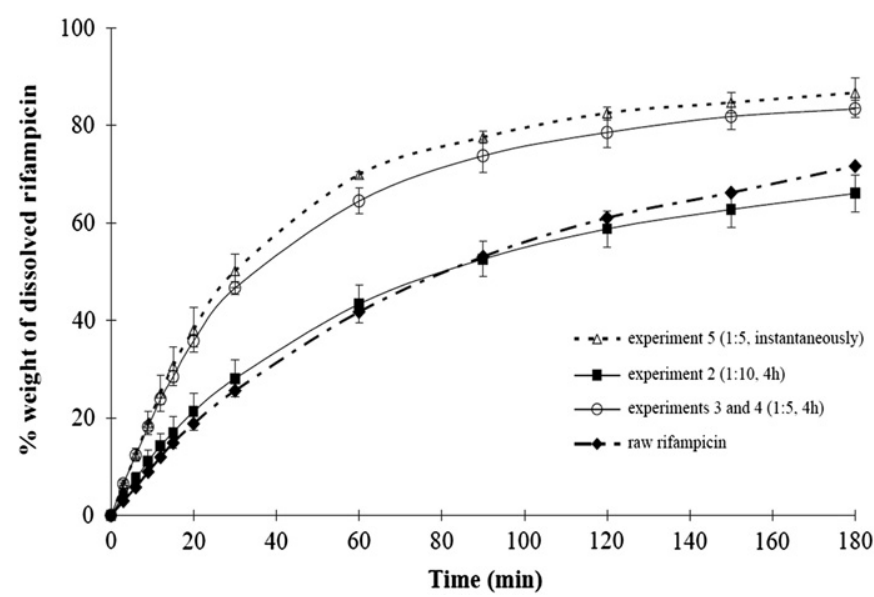

Fig. 8. Dissolution kinetic curves for rifampicin powders (testing conditions: water at $37{ }^{\circ} \mathrm{C}$ ). RIFA: raw rifampicin; experiments 2 to 5 precipitated from RIFA.

From the dissolution kinetic curves, it can be seen that the innovative method proposed in this study for drug precipitation from a RTIL solution seems to be an interesting way to improve the dissolution of the commercial crystals of rifampicin. The mass proportion between the solvent and the antisolvent could be the parameter to be adjusted to enhance dissolution of the nanosized powder. This statement is suggested by the higher drug dissolution rate obtained with RTIL:phosphate buffer of 1:5 in comparison to that obtained with a RTIL:phosphate buffer of 1:10 for the same process duration $(4 \mathrm{~h})$.

In addition, after being immersed for approximately 20 min into the dissolution medium, the nano-sized drug particles "instantaneously recovered" dissolved faster than those recovered after $4 \mathrm{~h}$ under agitation, probably due to a lower degree of particle agglomeration.

To sum up, after $45 \mathrm{~min}$, nearly $55 \%$ of the nanosized rifampicin was dissolved, while only $30 \%$ of the raw drug dissolved at that time. The increase in drug dissolution rate can be explained, in part, by the reduced particle size and by the enhanced specific surface area (in spite of the negative agglomeration effect).

It could also be verified in this study that the solubility (saturation concentration) of raw rifampicin and nanosized precipitated particles at $25 \pm 2{ }^{\circ} \mathrm{C}$ was $0.72 \mathrm{mg} / \mathrm{g}$ and $0.94 \mathrm{mg} / \mathrm{g}$, respectively. Reducing the particle size down to the submicron range and solid state amorphisation increase the solubility of rifampicin by $30 \%$.

\subsubsection{Antisolvent crystallization with the addition of stabilizers}

During the antisolvent precipitation process without any stabilizer, it was observed that the resultant nanosized particles of rifampicin tended to agglomerate together during precipitation.

Adsorption of polymeric excipients and surfactants to the surface of the newly formed particles can hinder their agglomeration through steric or electrostatic stabilization of the formed suspension $[34,35]$. This effect is predominant when the particles are smaller than $1 \mu \mathrm{m}$. To reduce this problem, hydroxypropyl methylcellulose (HPMC) was added to the antisolvent.

Although no differences in mean particle size, morphological aspects or physical state were observed among rifampicin particles formed with or without HPMC in the process, a significant difference was found on their dissolution kinetics (see Fig. 9). In fact, HPMC is a semi-synthetic ether derivative of cellulose that is widely used in many fields [36]. When present in the aqueous solution it may absorb on the surface of the formed hydrophobic drug particles to inhibit particle growth and aggregation as already reported [23]. The absorption of HPMC molecules on the surface of the nanosized particles of rifampicin freshly formed probably limits their agglomeration in the precipitation medium, as schematized in Fig. 10.

\subsubsection{Dissolution time and drug amount released}

The United States Pharmacopeia (USP) requires the dissolution of $80 \%$ of rifampicin within $45 \mathrm{~min}\left(Q_{45}\right)$ for an immediate release of oral solid dosage containing rifampicin. In our case, we performed powder dissolution as a mean to extrapolate this study for pure rifampicin powder in aqueous media. For the calculation of $Q_{45}$ (drug amount released after $45 \mathrm{~min}$ in the dissolution medium) and $t_{50 \%}$ (time to dissolve $50 \%$ of the drug), dissolution results were applied to the equation of Hixson-Crowell cube-root

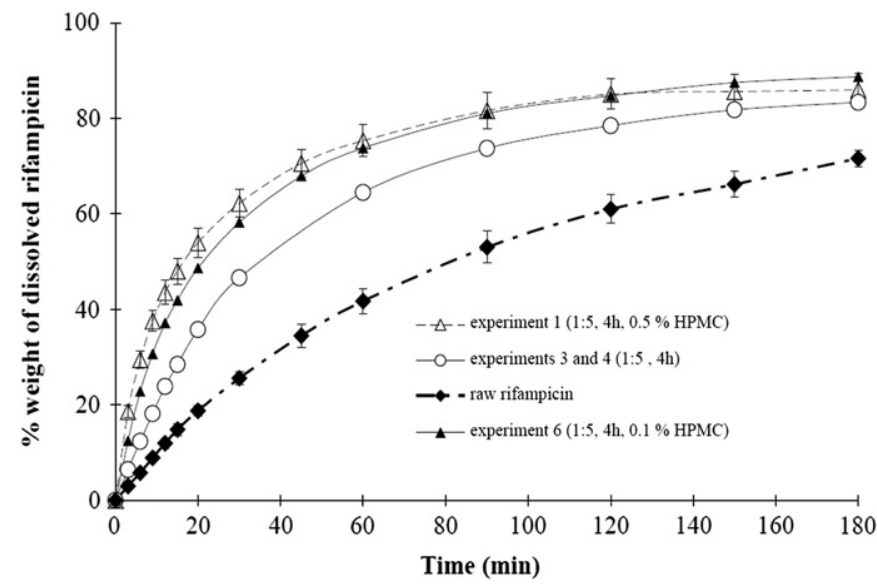

Fig. 9. Dissolution kinetic curves for rifampicin powders (testing conditions: water at $37^{\circ} \mathrm{C}$ ). RIFA: raw rifampicin; experiments $1,3,4$ and 6 precipitated from RIFA.

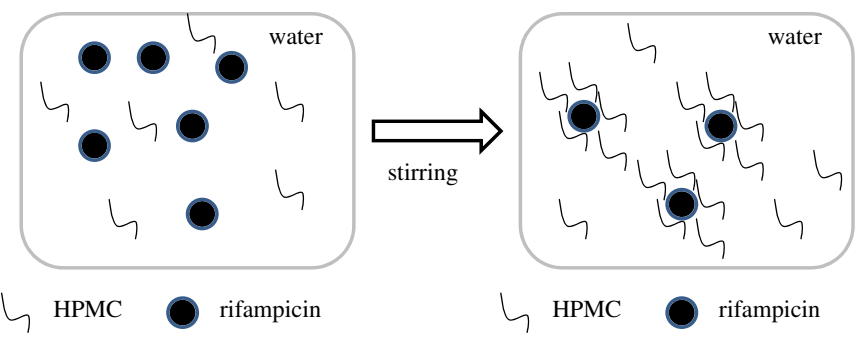

Fig. 10. Absorption of HPMC on the surface of rifamicin nanosized particles (modified from [23]). 
Table 3

$t_{50 \%}$ (time to dissolve $50 \%$ of the drug) and $Q_{45}$ (drug amount released after $45 \mathrm{~min}$ in the dissolution medium)

\begin{tabular}{lll}
\hline Experiment & $t_{50 \%}(\min )$ & $Q_{45}(\%)$ \\
\hline Raw rifampicin & 69 & 36 \\
3 and 4 & 29 & 68 \\
6 & 19 & 85 \\
7 & 17 & 87 \\
\hline
\end{tabular}

kinetic model (Eq. (1)), which is widely used by linearization of the dissolution profiles [37]

$\left(1-M_{t} / M_{0}\right)^{1 / 3}=1-k t$

where $k$ is the kinetic constant $\left(\mathrm{s}^{-1}\right), M_{t}$ is the mass of rifampicin dissolved in time $t$ and $M_{0}$ is the initial rifampicin mass in the RTIL solution. This model [38] describes the release from dosage forms, which show dissolution rate limitation and which do not dramatically change during the release process.

The dissolution data was plotted in accordance with HixsonCrowell cube root law (correlation coefficient $>0.997$ ) to determine the constant $k$. Although no real differences were observed between particle size and morphology of the precipitated powders, the data shown in Table 3 indicate that HPMC improves the dissolution kinetics of the nanosized rifampicin powder to the acceptable level required in USP $\left(Q_{45}>80 \%\right)$.

\section{Conclusion}

An innovative antisolvent precipitation method using RTIL (1-ethyl 3-methyl imidazolium methyl-phosphonate) as solvent for poorly water-soluble drugs such as rifampicin (BCS drug class II) was developed. The process can represent a simple and effective approach to produce submicron particles of poorly water-soluble drugs such as rifampicin, with a level of dissolution compliant to the United States Pharmacopeia. The next steps are to optimize the separation steps (solids separation, drying and solvent recycle), to better understand the RTILs solvency mechanism for rifampicin and to go deeply into the characterization of the physical state of the solid products.

Finally, the process proposed in this study will be applied to other BCS drugs (class II) to improve their solubility and dissolution kinetics in biorelevant media.

\section{Acknowledgment}

The authors acknowledge the Charcot Grant Programme (French Ministry of Foreign And European Affairs) that financed one year of doctoral thesis work to conduct research in pharmaceutical science at the RAPSODEE Centre (Université de Toulouse, Mines Albi, CNRS, France).

\section{References}

[1] C. Lipinski, Poor aqueous solubility-an industry wide problem in drug discovery, American Pharmaceutical Review 5 (2002) 82-85.

[2] S. Stegemann, F. Leveiller, D. Franchi, H. de Jong, H. Linden, When poor solubility becomes an issue: from early stage to proof of concept, European Journal of Pharmaceutical Sciences 31 (2007) 249-261.

[3] E. Merisko-Liversidge, G.G. Liversidge, E.R. Cooper, Nanosizing: a formulation approach for poorly-water-soluble compounds, European Journal of Pharmaceutical Science 18 (2003) 113-120.

[4] D. Douroumis, A. Fahr, Stable carbamazepine colloidal systems using the cosolvent technique, European Journal of Pharmaceutical Science 30 (2007) 367-374.
[5] R.H. Müller, K. Peters, Nanosuspensions for the formulation of poorly soluble drugs: I. Preparation by a size-reduction technique, International Journal of Pharmaceutics 160 (2008) 229-237.

[6] M. Perrut, J. Jung, F. Leboeuf, Enhancement of dissolution rate of poorly soluble active ingredients by supercritical fluid processes. Part I: micronization of neat particles, Journal of Pharmaceutics 288 (2005) 3-10.

[7] S.L. Raghavan, B. Kiepfer, A.F. Davis, S.G. Kazarian, J. Hadgraft, Membrane transport of hydrocortisone acetate from supersaturated solutions the role of polymers, International Journal of Pharmaceutics 221 (2001) 95-105.

[8] S.L. Raghavan, A. Trividic, A.F. Davis, J. Hadgraft, Crystallization of hydrocortisone acetate: influence of polymers, International Journal of Pharmaceutics 212 (2001) 213-222.

[9] X.X. Chen, J.M. Vaughn, M.J. Yacaman, R.O. Williams, K.P. Johnston, Rapid dissolution of high-potency danazol particles produced by evaporative precipitation into aqueous solution, Journal of Pharmaceutical Sciences 93 (2004) 1867-1878.

[10] C. Leuner, J. Dressman, Improving drug solubility for oral delivery using solid dispersions, European Journal of Pharmaceutics and Biopharmaceutics 50 (2000) 47-60.

[11] T. Vasconcelos, B. Sarmento, P. Costa, Solid dispersions as strategy to improve oral bioavailability of poor water soluble drugs, Drug Discovery Today 12 (2007) 1068-1075.

[12] C. Goddeeris, T. Willems, G. Van den Mooter, Formulation of fast disintegrating tablets of ternary solid dispersions consisting of TPGS 1000 and HPMC 2910 or PVPVA 64 to improve the dissolution of the anti-HIV drug UC 781 European Journal of Pharmaceutical Sciences 34 (2008) 293-302.

[13] J. Hecq, M. Deleers, D. Fanara, H. Vranckx, P. Boulanger, S. Le Lamer, K. Amighi, Preparation and in vitro/in vivo evaluation of nano-sized crystals for dissolution rate enhancement of ucb-35440-3, a highly dosed poorly water-soluble weak base, European Journal of Pharmaceutics Biopharmceutics 64 (3) (2006) 360-368.

[14] K.P. Krause, R.H. Müller, Production and characterisation of highly concentrated nanosuspensions by high pressure homogenization, International Journal of Pharmaceutics 214 (2001) 21-24.

[15] A. Tenorio, M.D. Gordillo, C. Pereyra, E.J. Martinez de la Ossa, Controlled submicro particle formation of ampicillin by supercritical antisolvent precipitation, Journal of Supercritical Fluids 40 (2007) 308-316.

[16] H. Chiou, L. Li, T. Hu, H.K. Chan, J.F. Chen, J. Yun, Production of salbutamol sulfate for inhalation by high-gravity controlled antisolvent precipitation, International Journal of Pharmaceutics 331 (2007) 93-98.

[17] J.Y. Zhang, Z.G. Shen, J. Zhong, T.T. Hu, J.F. Chen, Z.Q. Ma, J. Yun, Preparation of amorphous cefuroxime axetil nanoparticles by controlled nanoprecipitation method without surfactants, International Journal of Pharmaceutics 323 (2006) 153-160.

[18] Z. Wang, J.F. Chen, Y. Le, Z.G. Shen, J. Yun, Preparation of ultrafine beclomethasone dipropionate drug powder by antisolvent precipitation, Industrial and Engineering Chemistry Research 46 (2007) 4839-4845.

19] Y. Dong, W.K. Ng, S. Shen, S. Kim, R.B.H. Tan, Preparation and characterization of spironolactone nanoparticles by antisolvent precipitation, International Journal of Pharmaceutics 375 (2009) 84-88.

[20] N. Rasenack, H. Hartenhauer, B.W. Muller, Microcrystals for dissolution rate enhancement of poorly water-soluble drugs, International Journal of Pharmaceutics 254 (2003) 137-145.

[21] H. Zhao, J.X. Wang, Q.A. Wang, J.F. Chen, J. Yun, Controlled liquid antisolvent precipitation of hydrophobic pharmaceutical nanoparticles in a microchannel reactor, Industrial and Engineering Chemistry Research 46 (2007) 8229-8235.

[22] X.S. Li, J.X. Wang, Z.G. Shen, P.Y. Zhang, J.F. Chen, J. Yun, Preparation of uniform prednisolone microcrystals by a controlled microprecipitation method, International Journal of Pharmaceutics 342 (2007) 26-32.

[23] H.X. Zhang, J.X. Wang, Z.B. Zhang, Y. Le, Z.G. Shen, J.F. Chen, Micronization of atorvastatin calcium by antisolvent precipitation process, International Journal of Pharmaceutics 374 (2009) 2106-2113.

[24] X. Meng, Y. Chen, S.R. Chowdhury, D. Yang, S. Mitra, Stabilizing dispersions of hydrophobic drug molecules using cellulose ethers during antisolvent synthesis of micro-particulates, Colloids and Surfaces B: Biointerfaces 70 (2009) 7-14.

25] J.G. Huddleston, A.E. Visser, W.M Reichert, H.D. Willauer, G.A. Broker, R.D. Rogers, Characterization and comparison of hydrophilic and hydrophobic room temperature ionic liquids incorporating the imidazolium cation, Green Chemistry 3 (2001) 156-164.

[26] H. Mizuuch, V. Jaitely, S. Murdan, A.T. Florence, Room temperature ionic liquids and their mixtures: potential pharmaceutical solvents, European Journal of Pharmaceutical sciences. 33 (2008) 326-331.

[27] A. Fahr, X. Liu, Drug delivery strategies for poorly water-soluble drugs, Expert Opinion on Drug Delivery 4 (2007) 403-416.

[28] S.Q Henwood, M.M. de Villiers, W. Liebenberg. A.P. Lotter, Solubility and dissolution properties of generic rifampicin raw materials, Drug Development and Industrial Pharmacy 26 (2000) 403-408.

[29] S.K. Mehta, G. Kaur, K.K. Bhasin, Analysis of tween based microemulsion in the presence of TB drug rifampicin, Colloids and Surfaces B: Biointerfaces 60 (2007) 95-104.

[30] G. Pelizza, M. Nebuloni, P. Ferrari, G.G. Gallo, Polymorphism of rifampicin, Farmaco-Edizione Scientifica 32 (1977) 471-481.

[31] S. Agrawal, Y. Ashokraj, P.V. Bharatam, O. Pillai, R. Pachagnula, Solid-state characterization of rifampicin samples and its biopharmaceutic relevance, European Journal of Pharmaceutical Science 22 (2004) 27-144. 
[32] E. Reverchon, I. de Marco, G. Della Porta, Rifampicin microprticles production by supercritical antisolvent precipitation, International Journal of Pharmaceutics 243 (2002) 83-91.

[33] J.E.F. Reynolds, Martindale: the Extra Pharmacopoeia, 31st ed., The Royal Pharmaceutical Society of Great Britain, London, 1996, pp. 268.

[34] S.L. Law, J.B. Kayes, Adsorption of non-ionic water-soluble cellulose polymers at the solid-water interface and their effect on suspension stability, International Journal of Pharmaceutics 15 (1983) 251-260.

[35] A. Zimmermann, A. Millqvist-Fureby, M.R. Elema, T. Hansen, A. Müllertz, L. Hovgaard, Adsorption of pharmaceutical excipients onto microcrystals of siramesine hydrochloride: effects on physicochemical properties,
European Journal of Pharmaceutics and Biopharmaceutics 71 (2009) $109-116$

[36] E. Reverchon, G. Lamberti, A. Antonacci, Supercritical fluid assisted production of HPMC composite microparticles, The Journal of Supercritical Fluids 46 (2008) 185-196.

[37] X.Y. Su, R. Alkassas, A.L.W. Po, Statistical modelling of ibuprofen release from spherical lipophilic matrices, European Journal of Pharmaceutics and Biopharmaceutics 40 (1994) 73-76.

[38] A.W. Hixson, J.H. Crowell, Dependence of reaction velocity upon surface and agitation. I. Theoretical consideration, Industrial and Engineering Chemistry 23 (1931) 923-931. 